\title{
Fonction complexité associée à une application ergodique du tore
}

\author{
Jean-François Bertazzon \\ Laboratoire d'Analyse, Topologie et Probabilités, \\ Aix-Marseille Université, \\ Avenue de l'escadrille Normandie-Niémen. 13397 Marseille, France
}

17 août 2021

\begin{abstract}
In this article we give the optimal lower bound for the complexity function of a planar translation which induces an ergodic rotation of the torus $\mathbb{R}^{2} / \mathbb{Z}^{2}$. In addition, we give an explicit calculation of this complexity for the application $(x, y) \mapsto\left(x+1 / \phi^{2}, y+x-1 /\left(2 \phi^{3}\right)\right)$, where $\phi$ is the golden mean.
\end{abstract}

\section{RÉSUMÉ}

Nous proposons dans ce travail de minorer de manière optimale la fonction complexité associée à une translation du plan, qui induit une rotation ergodique du tore $\mathbb{R}^{2} / \mathbb{Z}^{2}$. De plus, nous donnons un calcul explicite de cette complexité pour l'application $(x, y) \mapsto\left(x+1 / \phi^{2}, y+\right.$ $\left.x-1 /\left(2 \phi^{3}\right)\right)$, où $\phi$ désigne le nombre d'or.

\section{Introduction}

Nous poursuivons dans ce travail, l'étude des interactions entre les systèmes dynamiques et les suites infinies avec un nombre fini de symboles. Nous nous concentrons principalement sur deux types de systèmes : les rotations $(x, y) \mapsto(x+\alpha, y+\beta)$ du tore $\mathbb{R}^{2} / \mathbb{Z}^{2}$, et les nilsystèmes affines, qui sont des extensions de rotations du type $(x, y) \mapsto(x+\alpha, y+x+\beta)$. Ces systèmes sont particulièrement intéressants car ils interviennent dans des problèmes délicats d'arithmétique, que ce soit le problème de généralisation de l'algorithme des fractions continues à la dimension 2 pour les rotations du tore, ou celui de la répartition des suites $\left(\alpha n^{2} \bmod 1\right)_{n}$ pour les nilsystèmes affines.

Il est alors relativement naturel de vouloir conjuguer ces systèmes avec des échanges de morceaux du plan. L'existence d'une telle conjugaison est une question délicate, liée à la recherche de partitions génératrices, sur laquelle nous ne nous attarderons pas : il existe des échanges de morceaux conjugués aux translations [6], et aux nilsystèmes affines [1]. Les échanges de morceaux se font dans un cas par translation et dans l'autre de manière affine.

Pour chaque système, il peut exister de nombreux échanges de morceaux qui lui sont conjugués. Nous pouvons associer à chaque échange de morceaux une fonction complexité qui compte le 
nombre de morceaux dans le raffinement de la partition formée par les morceaux initiaux. Entre deux échanges de morceaux, nous privilégierons celui qui a la fonction complexité la plus petite à celui qui a les morceaux les plus réguliers. Empiriquement, son étude donne plus de résultats sur le comportement du système.

Par exemple, pour tout couple $(\alpha, \beta)$ rationnellement indépendants, P. Arnoux, C. Mauduit, L. Shiokawa et J.-I. Tamura étudient dans 23 des échanges de morceaux polygonaux par translation, conjugués aux rotations du tore $(x, y) \mapsto(x+\alpha, y+\beta)$. Ils arrivent à calculer explicitement la fonction complexité conjecturée par G. Rauzy dans [6], et trouvent pour tout entier $n: p(n)=n^{2}+n+1$.

Cependant, pour certains couples $(\alpha, \beta)$, nous pouvons trouver des échanges de morceaux de complexité $p(n)=2 n+1$, dont le plus célèbre est celui qui échange les pièces du fractal de Rauzy. Bien que les morceaux soient à bords fractals, leurs échanges nous donnent beaucoup plus d'informations sur le système. Nous renvoyons à [4] pour mieux comprendre les interactions entre l'étude des suites infinies avec un nombre fini de symboles et les translations du cercle ou du tore.

Nous commençons par vérifier que cette complexité en $2 n+1$ est en fait optimale, puisque nous démontrons que tout échange de morceaux conjugué à une translation du plan est de complexité au moins $n \rightarrow 2 n+1$. Cependant, dans une deuxième partie, nous verrons que les nilsystèmes affines se comportent différemment. En effet, pour un exemple bien choisi, la complexité d'un de ces systèmes est égale à la complexité de la rotation du cercle dont il est l'extension $: n \rightarrow n+1$.

\section{Résultats principaux et notations}

Soit $T$ une application mesurable du plan $\mathbb{R}^{2}$ dans lui-même qui préserve la mesure de Lebesgue du plan $\mu$ et qui est $\mu$-presque sûrement $\mathbb{Z}^{2}$-périodique. C'est-à-dire

$$
\mu\left(\left\{\boldsymbol{x} \in \mathbb{R}^{2} ; T\left(\boldsymbol{x}+\mathbb{Z}^{2}\right) \not \subset\{T(\boldsymbol{x})\}+\mathbb{Z}^{2}\right\}\right)=0 .
$$

De telles applications induisent des applications $\bar{T}$ du tore $\mathbb{R}^{2} / \mathbb{Z}^{2}$ dans lui-même. Nous noterons $\bar{\mu}$ la mesure de Lebesgue du tore et $\overline{\mathcal{T}}$ le système dynamique $\left(\bar{T}, \mathbb{R}^{2} / \mathbb{Z}^{2}, \bar{\mu}\right)$.

Un échange de $m$ morceaux du plan $\mathcal{R}=(R, D, \mu)$ est dit adapté à l'application $T$ s'il vérifie les propriétés suivantes.

1) Le système dynamique qu'il engendre est équivalent en mesure au système $\overline{\mathcal{T}}$.

2) Le sous-ensemble du plan $D$ est la réunion presque sûrement disjointe de $m$ morceaux mesurables $\left(D_{n}\right)_{n \in\{1, \ldots, m\}}$ (i.e. $\forall(i, j) \in\{1, \ldots, m\}^{2}, \mu\left(D_{i} \cap D_{j}\right)=0$ ).

3) La mesure de l'ensemble des points $\boldsymbol{x}$ de $D$ tels qu'il existe un point $\boldsymbol{y}$ de $D$ distinct de $\boldsymbol{x}$, vérifiant $\boldsymbol{x}-\boldsymbol{y} \in \mathbb{Z}^{2}$, est nulle.

4) Pour tout point $\boldsymbol{x}$ de $D, R(\boldsymbol{x})=T(\boldsymbol{x})-\boldsymbol{n}_{i}$ si $\boldsymbol{x} \in D_{i}$, avec $\boldsymbol{n}_{i}=\left(n_{i}, m_{i}\right) \in \mathbb{Z}^{2}$.

En particulier, avec cette définition, nous n'imposons pas aux morceaux d'être bornés.

Considérons par exemple l'application $T:(x, y) \mapsto(2 x, 3 y)$. L'échange des 6 morceaux $D_{i, j}=$ $[i / 2,(i+1) / 2[\times[j / 3,(j+1) / 3[$ pour tout $(i, j) \in\{0,1\} \times\{0,1,2\}$, sur lesquels agit l'application $R(\boldsymbol{x})=T(\boldsymbol{x})-(i, j)$, si $\boldsymbol{x} \in D_{i, j}$, est adapté à $T$. 
Pour chaque échange de morceaux $\mathcal{R}$ adapté à $T$, le codage de l'orbite des points nous permet de définir un langage $\mathfrak{L}_{\mathcal{R}}$ de $\{1, \ldots, m\}^{*}$; ainsi qu'un système dynamique symbolique $\mathcal{S}_{\mathcal{R}}$. Nous noterons $p_{\mathcal{R}}$ la fonction complexité du langage $\mathcal{L}_{\mathcal{R}}$. Lorsque le système dynamique symbolique $\mathcal{S}_{\mathcal{R}}$ est conjugué en mesure au système dynamique $\overline{\mathcal{T}}$, nous dirons que l'échange de morceaux $\mathcal{R}$ est conjugué à $T$. Nous reviendrons plus longuement sur ces différents objets dans la section 3.

Lorsqu'il existe un échange de morceaux conjugué à $T$, nous pouvons définir la fonction complexité associée à l'application $T$ pour tout entier $n$, par

$$
p^{T}(n)=\inf \left\{p_{\mathcal{R}}(n) \text {, où } \mathcal{R} \text { est un échange de morceaux conjugué à } T\right\} .
$$

Par le théorème de Krieger, lorsque le système $\overline{\mathcal{T}}$ est d'entropie nulle pour la mesure $\bar{\mu}$, la fonction complexité associée à l'application $T$ est bien définie.

Dans la section 4, nous minorons la fonction complexité associée à une translation du plan.

Théorème 2.1. Si $T$ est une translation du plan $\mathbb{R}^{2}$, telle que $\overline{\mathcal{T}}$ soit ergodique, alors la fonction complexité associée à $T$ est bien définie et pour tout entier $n$, elle est supérieure à $2 n+1$.

Dans 6], G. Rauzy conjugue les rotations du tore avec des échanges de 3 morceaux du plan. Donc, pour une translation $T$ du plan, qui induit une translation ergodique du tore, la première valeur de la fonction complexité $p^{T}(1)$ est 3 . D'autre part, la translation $T$ conjuguée à l'échange des pièces du fractal de Rauzy est de complexité $n \rightarrow 2 n+1$. À l'heure actuelle, nous ne disposons que d'arguments constructifs pour majorer la complexité de toutes les translations du plan. Savoir si une translation du plan ergodique sur le tore est de complexité $n \rightarrow 2 n+1$ et si la complexité est atteinte pour tous les entiers par le même échange de morceaux, est une question encore ouverte, et à laquelle il semble très difficile d'apporter une réponse.

Dans la section5 nous construirons une suite d'échanges de morceaux, conjugués à un nilsystème affine bien choisi, dont la complexité décroît. Nous reprenons des idées utilisées par G. Rauzy, pour construire ce qui est appelé aujourd'hui le fractal de Rauzy. Mais contrairement à sa construction, la nôtre ne converge pas vers un «échange de morceaux bornés limite» (cf. remarque 1). Une des conséquences de ce résultat est qu'il n'y a donc pas, a priori, d'échanges de morceaux bornés privilégiés du point de vue de la complexité pour s'intéresser aux nilsystèmes affines.

Théorème 2.2. Notons $T_{\phi}$ l'application définie par

$$
T_{\phi}(x, y)=\left(x+1 / \phi^{2}, y+x-1 /\left(2 \phi^{3}\right)\right) \text {, où } \phi \text { est le nombre d'or. }
$$

Pour tout entier $M$ fixé, il existe un échange de morceaux $\mathcal{R}_{M}$ conjugué à $\overline{\mathcal{T}}_{\phi}$ tel que pour tout entier $1 \leq k \leq M, p_{\mathcal{R}_{M}}(k)=k+1$. C'est-à-dire, pour tout entier $n, p^{T_{\phi}}(n)=n+1$.

\section{Objets symboliques}

Nous reprenons les notations de l'introduction. Soit $T$ une application mesurable du plan $\mu$ presque sûrement $\mathbb{Z}^{2}$-périodique et qui préserve la mesure de Lebesgue. Fixons $\mathcal{R}$ un échange de $m$ morceaux conjugué à $T$. 
Pour $\mu$-presque tout point $\boldsymbol{x} \in D, C(\boldsymbol{x})$ désigne la suite d'éléments de $\{1, \ldots, m\}^{\mathbb{N}}$ dont le $n$-ième terme est $i$ si $R^{n}(\boldsymbol{x}) \in D_{i}$. Il est relativement clair que cette application de codage $C$ est mesurable. Nous noterons $S_{\mathcal{R}}$ l'ensemble des suites obtenues de cette manière et $\mu_{\mathcal{R}}$ la mesure de probabilité sur $S_{\mathcal{R}}$ qui provient de la mesure de Lebesgue $\mu$ via $C$ (c'est-à-dire pour tout ensemble mesurable $\left.A, \mu_{\mathcal{R}}(A)=\mu\left(C^{-1} A\right)\right)$. Le système dynamique symbolique $\mathcal{S}_{\mathcal{R}}$ associé à $\mathcal{R}$ est le triplet $\left(\mathcal{S}, S_{R}, \mu_{\mathcal{R}}\right)$, où $\mathcal{S}$ désigne le décalage classique. De cette manière, il est clair que le système $\mathcal{S}_{\mathcal{R}}$ est un facteur mesuré du système $\overline{\mathcal{T}}$ et que l'application de codage $C$ est l'application facteur associée. Les codages ainsi obtenus sont les codages «naturels» décrits par V. Berthé, S. Ferenczi et L.Q. Zamboni dans [4].

Notons $\{1, \ldots, m\}^{*}$ l'ensemble des mots à valeur dans $\{1, \ldots, m\}$ finis ou infinis. La longueur d'un mot $u \in\{1, \ldots, m\}^{*}$ sera notée $|u| \in \mathbb{N} \cup\{+\infty\}$. Soient $v \in\{1, \ldots, m\}^{\mathbb{N}}$ et $u \in\{1, \ldots, m\}^{*}$. Le mot $u$ est un facteur de $v$ s'il existe un entier $m$ tel que pour tout entier $n \leq|u|, u_{n}=v_{m+n}$. Un langage est un sous-ensemble de $\{0,1\}^{*}$. Il est dit factoriel si pour tout mot $v$ du langage, tout facteur $u$ de $v$ appartient au langage. Nous noterons $\mathcal{L}(\mathfrak{L})$ le langage factoriel engendré par un langage $\mathfrak{L}$. Nous définissons le langage $\mathfrak{L}_{\mathcal{R}}$ associé à l'échange de morceaux $\mathcal{R}$, par $\mathfrak{L}_{\mathcal{R}}=\mathcal{L}\left(S_{\mathcal{R}}\right)$. La fonction complexité $p_{\mathcal{R}}$ associée au langage $\mathfrak{L}_{\mathcal{R}}$ est définie pour tout entier $n$ par :

$$
p_{\mathcal{R}}(n)=\#\left\{u \in \mathfrak{L}_{\mathcal{R}} \text { tel que }|u|=n\right\} .
$$

Ces définitions ne sont sûrement pas les meilleures pour une étude générale des applications du tore, mais elles suffiront amplement pour s'intéresser aux classes de systèmes très particulières que sont les translations et les nilsystèmes affines.

Nous rappelons également le résultat suivant de P. Halmos qui permet de déterminer si le système symbolique associé à un échange de morceaux adapté à $T$ est conjugué en mesure au système $\overline{\mathcal{T}}$.

Proposition 3.1 (P. Halmos). Soit $T$ une application du plan $\mu$-presque sûrement $\mathbb{Z}^{2}$-périodique, qui préserve la mesure de Lebesgue et telle que le système $\overline{\mathcal{T}}$ soit ergodique. Soit $\mathcal{R}$ un échange de $m$ morceaux du plan adapté à $T$. Alors, les assertions suivantes sont équivalentes.

1) L'échange de morceaux $\mathcal{R}$ est conjugué à $T$.

2) Pour toute suite $\left(u_{n}\right) \in\{1, \ldots, m\}^{\mathbb{N}}$,

$$
\mu\left(\bigcap_{k=1}^{N} R^{-k} D_{u_{k}}\right) \underset{N \rightarrow+\infty}{\longrightarrow} 0 .
$$

3) La mesure de Lebesgue de l'ensemble $\{\boldsymbol{x} \in D$ tel qu'il existe $\boldsymbol{y} \in D \backslash\{\boldsymbol{x}\}$ tel que $C(\boldsymbol{x})=C(\boldsymbol{y})\}$ est nulle.

Proposition 3.2. Soit $\overline{\mathcal{T}}$ un système dynamique défini sur le tore $\mathbb{R}^{2} / \mathbb{Z}^{2}$. Fixons $T$ un relèvement de $\bar{T}$ défini dans le plan et supposons qu'il existe un échange de morceaux conjugué à $T$. Nous supposons, de plus, que le système $\overline{\mathcal{T}}$ est totalement ergodique (c'est-à-dire que toutes ses puissances $\overline{\mathcal{T}}^{k}=\left(\bar{T}^{k}, \mathbb{R}^{2} / \mathbb{Z}^{2}, \bar{\mu}\right)$ sont ergodiques $)$. Alors, la fonction complexité associée à $T$ existe et crồt strictement. En particulier, pour tout entier $n$ elle est supérieure ou égale à $n+1$.

de la proposition 3.2. Fixons un système $\overline{\mathcal{T}}$ satisfaisant les hypothèses de la proposition. Supposons alors qu'un des échanges de morceaux conjugués à $T$ ait une $n$-ième complexité égale à $j$ et une $(n+1)$-ième complexité encore égale à $j$. Cela veut donc dire que la partition initiale se raffine 
en $j$ morceaux $P_{1}, P_{2}, \ldots P_{j}$, lorsque l'on s'interesse à ses $(n-1)$-ièmes pré-images. Puisque la $(n+1)$-ième complexité est encore égale à $j$, cela signifie que la partition $P_{1}, \ldots P_{j}$ ne se raffine pas. Par ergodicité, l'application $\boldsymbol{x} \mapsto i \in\{1, \ldots, j\}$ si $\boldsymbol{x} \in P_{i}$ définit un facteur mesuré. Or, puisque le système est totalement ergodique, il n'admet pas de facteur fini. La fonction $p^{T}$ est donc strictement croissante.

On conclut alors en remarquant qu'il faut échanger au moins deux morceaux pour conjuguer le système ergodique du tore $\overline{\mathcal{T}}$ avec le système symbolique engendré par l'échange de morceaux.

\section{Preuve du théorème 2.1}

Lemme 4.1. Si $T$ est une translation du plan telle que $\overline{\mathcal{T}}$ soit ergodique, alors $p^{T}(1) \geq 3$.

Preuve du lemme 4.1. Fixons deux réels $r$ et $s$ tels que la translation $T:(x, y) \mapsto(x+r, y+s)$ soit ergodique sur le tore $\mathbb{R}^{2} / \mathbb{Z}^{2}$ (c'est-à-dire pour tout couple d'entiers $(n, m)$ non-nuls simultanément, $n r+m s \notin \mathbb{Z})$.

Supposons que l'application $T$ soit conjuguée à un échange de deux morceaux $D_{1}$ et $D_{2}$, de mesure strictement positive (respectivement $\alpha_{1}$ et $\alpha_{2}$ ). Puisque $\mu\left(D_{1} \cup D_{2}\right)=1$, et que $\mu\left(D_{1} \cap D_{2}\right)=$ 0 , on a immédiatement $\alpha_{1}+\alpha_{2}=1$. Par le théorème ergodique de Birkhoff et de récurrence de Poincaré, il existe un point récurent $\boldsymbol{x}_{0}=\left(x_{0}, y_{0}\right)$ de $D$, tel que

$$
\frac{1}{N} \sum_{n=0}^{N-1} \mathbb{1}_{R^{-n} D_{1}}\left(\boldsymbol{x}_{0}\right) \rightarrow \alpha_{1} \text { et } \frac{1}{N} \sum_{n=0}^{N-1} \mathbb{1}_{R^{-n} D_{2}}\left(\boldsymbol{x}_{0}\right) \rightarrow \alpha_{2}
$$

Traduisons maintenant la condition de récurrence imposée sur le point $\boldsymbol{x}_{0}$. Pour tout entier $N$ :

$$
R^{N}\left(\boldsymbol{x}_{0}\right)=\left(\begin{array}{c}
x_{0}+N r-n_{1} \sum_{n=0}^{N-1} \mathbb{1}_{R^{-n} D_{1}}\left(\boldsymbol{x}_{0}\right)-n_{2} \sum_{n=0}^{N-1} \mathbb{1}_{R^{-n} D_{2}}\left(\boldsymbol{x}_{0}\right) \\
y_{0}+N s-m_{1} \sum_{n=0}^{N-1} \mathbb{1}_{R^{-n} D_{1}}\left(\boldsymbol{x}_{0}\right)-m_{2} \sum_{n=0}^{N-1} \mathbb{1}_{R^{-n} D_{2}}\left(\boldsymbol{x}_{0}\right)
\end{array}\right) .
$$

Puisque le point $\boldsymbol{x}_{0}$ est récurent, il existe une sous-suite croissante d'entiers $\left(N_{k}\right)_{k}$ telle que $R^{N_{k}}\left(\boldsymbol{x}_{0}\right) / N_{k}$ converge vers $\mathbf{0}$. Ce qui nous amène aux équations :

$$
r=n_{1} \alpha_{1}+n_{2} \alpha_{2} \text { et } s=m_{1} \alpha_{1}+m_{b} \alpha_{2}
$$

Nous trouvons donc :

$$
r=n_{1}+\alpha_{2}\left(n_{2}-n_{1}\right) \text { et } s=m_{1}+\alpha_{2}\left(m_{2}-m_{1}\right)
$$

En remarquant que les quantités $n_{2}-n_{1}$ et $m_{2}-m_{1}$ sont non nulles (sinon $r$ ou $s$ serait entier), nous trouvons alors que $\left(m_{2}-m_{1}\right) r-\left(n_{2}-n_{1}\right) s \in \mathbb{Z}$, ce qui est absurde.

Proposition 4.2. Si $T$ est une translation du plan telle que $\overline{\mathcal{T}}$ soit ergodique, alors $p^{T}(2) \geq 5$.

Preuve de la proposition 4.2. Fixons deux réels $r$ et $s$ tels que la translation $T:(x, y) \mapsto(x+r, y+s)$ soit ergodique sur le tore $\mathbb{R}^{2} / \mathbb{Z}^{2}$, et $\mathcal{R}$ un échange de trois morceaux conjugué à $T$, tel que $p_{\mathcal{R}}(2) \leq 4$. 
Par la proposition 3.2 , la fonction complexité $p_{\mathcal{R}}$ est une fonction strictement croissante. De plus, nous avons vu dans le lemme 4.1 que $p_{\mathcal{R}}(1)=3$. Il nous faut donc montrer qu'il est impossible d'avoir un échange de morceaux $\mathcal{R}$ conjugué à $T$ tel que $p_{\mathcal{R}}(1)=3$ et $p_{\mathcal{R}}(2)=4$.

Supposons qu'il existe $\mathcal{R}$, un échange de trois morceaux $D_{1}, D_{2}$ et $D_{3}$ de mesures respectives $\alpha_{1}, \alpha_{2}$ et $\alpha_{3}$, adapté à $T$, tel que $p_{\mathcal{R}}(2)=4$. Nécessairement, un seul des trois morceaux se raffine quand on s'interesse à la pré-image de la partition formée par les trois morceaux $D_{1}, D_{2}$ et $D_{3}$. Supposons donc que le morceau $D_{1}$ se raffine en deux morceaux $D_{1}^{(1)}$ et $D_{1}^{(2)}$, de mesure $\alpha_{1}^{(1)}$ et $\alpha_{1}^{(2)}$. En reprenant les arguments de la preuve du lemme 4.1 nous pouvons montrer qu'il existe six entiers $n_{1}, n_{2}, n_{3}, m_{1}, m_{2}$ et $m_{3}$, tels que :

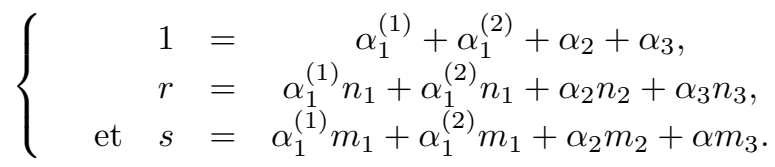

Puisque les morceaux $D_{2}$ et $D_{3}$ ne se raffinent pas, il existe $(i, j) \in\{1,2,3\}^{2}$ tels que $R\left(D_{2}\right) \subset D_{i}$ et $R\left(D_{3}\right) \subset D_{j}$. Remarquons immédiatement que par ergodicité, et puisque $R$ préserve la mesure, il est impossible que $R\left(D_{2}\right) \subset D_{2}$, ou que $R\left(D_{3}\right) \subset D_{3}$. Quitte à renuméroter $D_{1}^{(1)}$ et $D_{1}^{(2)}$, ou 2 et 3 , il n'y a que trois cas possibles :

\begin{tabular}{|l||l|l|l|l|}
\hline Cas 1 & $R\left(D_{1}^{(2)}\right) \subset D_{3}$ & $R\left(D_{1}^{(1)}\right) \subset D_{2}$ & $R\left(D_{2}\right) \subset D_{1}$ & $R\left(D_{3}\right) \subset D_{1}$ \\
\hline Cas 2 & $R\left(D_{1}^{(2)}\right) \subset D_{3}$ & $R\left(D_{1}^{(1)}\right) \subset D_{1}$ & $R\left(D_{2}\right) \subset D_{1}$ & $R\left(D_{3}\right) \subset D_{2}$ \\
\hline Cas 3 & $R\left(D_{1}^{(2)}\right) \subset D_{3}$ & $R\left(D_{1}^{(1)}\right) \subset D_{2}$ & $R\left(D_{2}\right) \subset D_{1}$ & $R\left(D_{3}\right) \subset D_{2}$ \\
\hline
\end{tabular}

Nous allons étudier chacun des cas et vérifier qu'ils mènent tous à une absurdité. Pour cela, nous allons traduire les répercussions de ces inclusions sur les valeurs de $\alpha_{1}^{1}, \alpha_{1}^{2}, \alpha_{2}$ et $\alpha_{3}$; en utilisant le fait que l'application $R$ préserve la mesure de Lebesgue.

Premier cas. Puisque $R\left(D_{3}\right) \cup R\left(D_{2}\right) \subset D_{1}$, alors $\mu\left(R\left(D_{3}\right) \cup R\left(D_{2}\right)\right) \leq \alpha_{1}=\alpha_{1}^{(1)}+\alpha_{1}^{(2)}$. De plus, $\mu\left(R\left(D_{3}\right) \cup R\left(D_{2}\right)\right) \leq \alpha_{2}+\alpha_{3}$. De même, $\alpha_{1}^{(1)} \leq \alpha_{2}$ et $\alpha_{1}^{(2)} \leq \alpha_{3}$. Puisque $\alpha_{1}^{(1)}+\alpha_{1}^{(2)}+\alpha_{2}+\alpha_{3}=1$, alors nécessairement :

$$
\alpha_{2}+\alpha_{3}=\alpha_{1}^{(1)}+\alpha_{1}^{(2)}, \alpha_{1}^{(1)}=\alpha_{2} \text { et } \alpha_{1}^{(2)}=\alpha_{3} .
$$

Le système 2 devient alors :

$$
1 / 2=\alpha_{2}+\alpha_{3}, r=\alpha_{2}\left(n_{1}+n_{b}\right)+\alpha_{3}\left(n_{1}+n_{3}\right) \text { et } s=\alpha_{2}\left(m_{1}+m_{2}\right)+\alpha_{3}\left(m_{1}+m_{3}\right) .
$$

Comme nous l'avons vu dans la preuve du lemme 4.1, les réels $r$ et $s$ sont alors rationnellement dépendants et le système $\overline{\mathcal{T}}$ n'est donc pas ergodique. C'est absurde.

Deuxième cas. L'étude des inclusions nous amène aux conditions suivantes :

$$
\alpha_{3}=\alpha_{2}, \alpha_{2}+\alpha_{1}^{(1)}=\alpha_{1}=\alpha_{1}^{(1)}+\alpha_{1}^{(2)} \text { et } \alpha_{1}^{(2)}=\alpha_{3} .
$$

Donc $\alpha_{3}=\alpha_{2}=\alpha_{1}^{(2)}$ et le système 2 devient alors :

$$
1=\alpha_{1}^{(1)}+3 \alpha_{3}, r=\alpha_{1}^{(1)} n_{1}+\alpha_{3}\left(n_{1}+n_{2}+n_{3}\right) \text { et } s=\alpha_{1}^{(1)} m_{1}+\alpha_{3}\left(m_{1}+m_{2}+m_{3}\right) .
$$


Comme précédemment, cela nous amène à une absurdité.

Troisième cas. L'étude des inclusions impose les conditions suivantes :

$$
\alpha_{3}+\alpha_{1}^{(1)}=\alpha_{2}, \alpha_{2}=\alpha_{1}=\alpha_{1}^{(1)}+\alpha_{1}^{(2)} \text { et } \alpha_{1}^{(2)}=\alpha_{3} .
$$

Donc $\alpha_{3}=\alpha_{2}=\alpha_{1}^{(2)}$ et le système 2 s'écrit :

$$
1=2 \alpha_{2}+\alpha_{3}, r=\alpha_{2}\left(n_{1}+n_{2}\right)+\alpha_{3} n_{3} \text { et } s=\alpha_{2}\left(m_{1}+m_{2}\right)+\alpha_{3} m_{3} .
$$

Encore comme précédemment, cela nous amène à une contradiction.

Preuve du théorème 2.1. Fixons deux réels $r$ et $s$ tels que la translation $T:(x, y) \mapsto(x+r, y+s)$ soit ergodique sur le tore $\mathbb{R}^{2} / \mathbb{Z}^{2}$, ainsi qu'un échange de morceaux $\mathcal{R}$ conjugué à $T$. Fixons également un entier $n$ jusqu'auquel le résultat du théorème est vrai et montrons que le résultat persiste jusqu'au cran $n+1$

Comme dans la preuve de la proposition 4.2, il y a des situations trivialement dégénérées. Supposons par la suite que pour tout entier $i \in\{1, \ldots, n\}, p_{\mathcal{R}}(i)=2 i+1$, et montrons qu'il est impossible que $p_{\mathcal{R}}(n+1)$ soit égale à $2 n+2$.

Le $(n-1)$-ième raffinement des morceaux initiaux est donc composé de $2 n+1$ morceaux notés $\left(D_{1}, \ldots, D_{2 n+1}\right)$ de mesure $\alpha_{1}, \ldots, \alpha_{2 n+1}$. Un seul de ces $2 n+1$ morceaux se raffine quand on s'interesse à la $n$-ième pré-image de la partition formée par les trois morceaux initiaux. Supposons donc que le morceau $D_{1}$ se raffine en deux morceaux $D_{1}^{(1)}$ et $D_{1}^{(2)}$, de mesure $\alpha_{1}^{(1)}$ et $\alpha_{1}^{(2)}$. Comme précédemment, pour tout $i \in\{2, \ldots, 2 n+1\}$, il existe $j \in\{1, \ldots, 2 n+1\}$ tel que $R\left(D_{i}\right) \subset D_{j}$.

Nous pouvons utiliser les arguments du lemme 4.1 pour monter qu'il existe des entiers $\left(n_{i}\right)_{i=1 \ldots 2 n+1}$ et $\left(m_{i}\right)_{i=1 \ldots 2 n+1}$ tels que :

$$
\left\{\begin{aligned}
1 & =\alpha_{1}^{(1)}+\alpha_{1}^{(2)}+\alpha_{2}+\alpha_{3}+\cdots+\alpha_{2 n+1} \\
r & =\alpha_{1}^{(1)} n_{1}+\alpha_{1}^{(2)} n_{1}+\alpha_{2} n_{2}+\cdots+\alpha_{2 n+1} n_{2 n+1}, \\
\text { et } s & =\alpha_{1}^{(1)} m_{1}+\alpha_{1}^{(2)} m_{1}+\alpha_{2} m_{2}+\cdots+\alpha_{2 n+1} m_{2 n+1} .
\end{aligned}\right.
$$

Quitte à réindexer les indices, deux cas peuvent alors se produire :

$$
\begin{array}{ll}
\text { Cas 1: } & R\left(D_{1}^{(1)}\right) \subset D_{1} \text { et } R\left(D_{1}^{(2)}\right) \subset D_{2} . \\
\text { Cas 2: } & R\left(D_{1}^{(1)}\right) \subset D_{2} \text { et } R\left(D_{1}^{(2)}\right) \subset D_{3} .
\end{array}
$$

Premier cas. C'est le cas le plus simple : une seule zone peut rentrer dans la zone $D_{1}$. Quitte à réindexer les indices, on peut voir que puisque le système $\mathcal{R}$ est ergodique, il n'est pas possible que la réunion de certaines zones soit envoyée dans elle-même. Donc $R\left(D_{2}\right) \subset D_{3}, R\left(D_{3}\right) \subset D_{4}$, $\ldots, R\left(D_{2 n}\right) \subset D_{2 n+1}$ et $R\left(D_{2 n+1}\right) \subset D_{1}$. Ce qui nous amène aux contraintes suivantes sur les coefficients $\alpha_{i}$ :

$$
\alpha_{1}^{(1)}+\alpha_{2 n+1}=\alpha_{1}^{(1)}+\alpha_{1}^{(2)}, \alpha_{1}^{(2)}=\alpha_{2}=\alpha_{3}, \ldots \ldots, \alpha_{2 n-1}=\alpha_{2 n} \text { et } \alpha_{2 n}=\alpha_{2 n+1} .
$$

Soit encore $: \alpha_{1}^{(2)}=\alpha_{2}=\alpha_{3}=\cdots=\alpha_{2 n+1}$. Le système 3 se réécrit donc :

$$
1=\alpha_{1}^{(1)}+(2 n+1) \alpha_{1}^{(2)}, r=\alpha_{1}^{(1)} n_{1}+\alpha_{1}^{2} N \text { et } s=\alpha_{1}^{(1)} m_{1}+\alpha_{1}^{2} M,
$$


où $N=\sum_{i=1}^{2 n+1} n_{i}$ et $M=\sum_{i=1}^{2 n+1} m_{i}$. On conclut alors comme la preuve de la proposition 4.2 ,

Deuxième cas. C'est plus compliqué. En effet, deux zones peuvent rentrer dans la zone $D_{1}$. Comme dans la preuve de la proposition 4.2 , quitte à réindexer les indices, deux sous-cas peuvent se produire.

$$
\begin{array}{cl}
\text { Premier sous-cas : } & D_{1}^{(1)} \rightarrow D_{2} \rightarrow D_{4}--\rightarrow D_{2 k} \longrightarrow D_{2 k+2--\rightarrow} D_{2 n+1} \rightarrow D_{1} . \\
& D_{1}^{(2)} \rightarrow D_{3} \rightarrow D_{5--} \rightarrow D_{2 k+1} \longrightarrow
\end{array}
$$

Deuxième sous-cas : $\quad D_{1}^{(1)} \rightarrow D_{2} \rightarrow D_{4}--\rightarrow D_{2 k} \longrightarrow D_{1}$,

$$
D_{1}^{(2)} \rightarrow D_{3} \rightarrow D_{5}-\rightarrow D_{2 k+1} \longrightarrow D_{2 k+2}--\rightarrow D_{2 n+1} \rightarrow D_{1} .
$$

Premier sous-cas. Les conditions sur les coefficients sont :

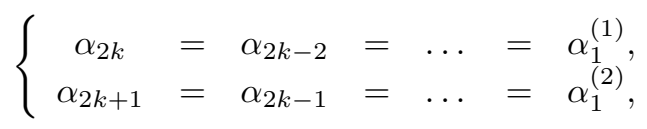

et $\alpha_{2 k}+\alpha_{2 k+1}=\alpha_{2 k+2}=\cdots=\alpha_{2 n+1}=\alpha_{1}=\alpha_{1}^{(1)}+\alpha_{1}^{(2)}$.

Posons $N_{1}=n_{1}+\sum_{i=1}^{k} n_{2 i}+\sum_{i=2 k+2}^{2 n+1} n_{i}, N_{2}=n_{1}+\sum_{i=1}^{k} n_{2 i+1}+\sum_{i=2 k+2}^{2 n+1} n_{i}, M_{1}=m_{1}+\sum_{i=1}^{k} m_{2 i}+\sum_{i=2 k+2}^{2 n+1} m_{i}$, et $M_{2}=m_{1}+\sum_{i=1}^{k} m_{2 i+1}+\sum_{i=2 k+2}^{2 n+1} m_{i}$. Le système 3 devient donc :

$$
\left\{\begin{array}{rrrr}
1 & = & & (2 n-k+1) \alpha_{1}^{(1)}+(2 n-k+1) \alpha_{1}^{(2)}, \\
r & = & \alpha_{1}^{(1)} N_{1}+\alpha_{1}^{(2)} N_{2}, \\
\text { et } s= & \alpha_{1}^{(1)} M_{1}+\alpha_{1}^{(2)} M_{2} .
\end{array}\right.
$$

On conclut alors comme précédemment.

Deuxième sous-cas. Les conditions sur les coefficients sont :

$$
\left\{\begin{array}{l}
\alpha_{2 k}=\alpha_{2 k-2}=\cdots=\alpha_{2}=\alpha_{1}^{(1)} \\
\alpha_{2 n+1}=\alpha_{2 n}=\cdots=\alpha_{2 k+1}=\cdots=\alpha_{3}=\alpha_{1}^{(2)}, \\
\alpha_{2 k}+\alpha_{2 n+1}=\alpha_{1}=\alpha_{1}^{(1)}+\alpha_{1}^{(2)}
\end{array}\right.
$$

En posant alors $N_{1}=n_{1}+\sum_{i=1}^{k} n_{2 i}, N_{2}=n_{1}+\sum_{i=1}^{k} n_{2 i+1}+\sum_{i=2 k+2}^{2 n+1} n_{i}, M_{1}=m_{1}+\sum_{i=1}^{k} m_{2 i}$, et $M_{2}=m_{1}+\sum_{i=1}^{k} m_{2 i+1}+\sum_{i=2 k+2}^{2 n+1} m_{i}$, le système 3 s'écrit :

$$
\left\{\begin{aligned}
1 & =\alpha_{1}^{(1)}(k+1)+\alpha_{1}^{(2)}(2 n-k+1) \\
r & =\alpha_{1}^{(1)} N_{1}+\alpha_{1}^{(2)} N_{2} \\
\text { et } s & =\alpha_{1}^{(1)} M_{1}+\alpha_{1}^{(2)} M_{2}
\end{aligned}\right.
$$


On conclut encore comme précédemment.

\section{Preuve du théorème 2.2}

Notons $\sigma$ la substitution de Fibonacci définie par

$$
\sigma: 1 \mapsto 12 \text { et } 2 \mapsto 1 \text {. }
$$

La preuve du théorème se déduit des deux résultats suivants.

Proposition 5.1. Soit $\mathcal{R}$ un échange de deux morceaux $D_{1}$ et $D_{2}$ conjugué à $T_{\phi}$, défini par

$$
R(\boldsymbol{x})=T_{\phi}(\boldsymbol{x}) \text { si } \boldsymbol{x} \in D_{1} \text {, et } R(\boldsymbol{x})=T_{\phi}(\boldsymbol{x})-(1,0) \text { si } \boldsymbol{x} \in D_{2} \text {. }
$$

En notant $\mathfrak{p}$ la projection de $\mathbb{R}^{2}$ dans $\mathbb{R}$ définie par $\mathfrak{p}(x, y)=x$, nous supposons de plus l'existence d'un réel $z$ tel que $\left.\left.\mathfrak{p}\left(D_{1}\right) \subset\right] z-1, z+1 / \phi^{2}\right]$ et $\left.\mathfrak{p}\left(D_{2}\right) \subset\right] z-1 / \phi^{2}, z+1 / \phi^{2}[$.

Alors, il existe alors un échange de deux morceaux $\mathcal{R}^{\prime}$ conjugué à $\mathcal{T}_{\phi}$, de même nature que $\mathcal{R}$, tel que :

$$
\mathfrak{L}_{\mathcal{R}^{\prime}}=\mathcal{L}\left(\sigma\left(\mathfrak{L}_{\mathcal{R}}\right)\right) .
$$

Proposition 5.2. Il existe $\mathcal{R}^{(1)}$, un échange de deux morceaux bornés, connexes et simplement connexes $D_{1}^{(1)}$ et $D_{2}^{(1)}$ conjugué à $T_{\phi}$. Les mesures de $D_{1}^{(1)}$ et $D_{2}^{(1)}$ sont respectivement $1 / \phi$ et $1 / \phi^{2}$. L'application $R^{(1)}$ est définie par:

$$
R^{(1)}(\boldsymbol{x})=T_{\phi}(\boldsymbol{x}) \text { si } \boldsymbol{x} \in D_{1}^{(1)} \text {, et } R^{(1)}(\boldsymbol{x})=T_{\phi}(\boldsymbol{x})-(1,0) \text { si } \boldsymbol{x} \in D_{2}^{(1)} .
$$

De plus, il existe un réel $z$ tel que:

$$
\left.\left.\left.\mathfrak{p}\left(D_{1}^{(1)}\right) \subset\right] z-1, z+1 / \phi^{2}\right] \text { et } \mathfrak{p}\left(D_{2}^{(1)}\right) \subset\right] z-1 / \phi^{2}, z+1 / \phi^{2}[.
$$

Preuve de la proposition 5.1. Nous allons construire explicitement l'échange de morceaux $\mathcal{R}^{\prime}$. Pour cela, nous définissons une application $\psi: \mathbb{R}^{2} \longrightarrow \mathbb{R}^{2}$ par

$$
\psi(x, y)=\left(-\phi x,-y+\alpha x^{2}+\beta x+\gamma\right),
$$

où $\alpha, \beta$ et $\gamma$ sont des paramètres réels que nous fixerons plus tard. L'application $\psi$ est bijective et son inverse est :

$$
\psi^{-1}(x, y)=\left(-x / \phi,-y+\alpha x^{2} / \phi^{2}-\beta x / \phi+\gamma\right) .
$$

Nous posons alors :

$$
D_{1}^{\prime}=\psi^{-1}(D)=\psi^{-1}\left(D_{1} \cup D_{2}\right), D_{2}^{\prime}=T_{\phi}\left(\psi^{-1}\left(D_{1}\right)\right) \text { et } D^{\prime}=D_{1}^{\prime} \cup D_{2}^{\prime} .
$$

Nous considérons l'application $R^{\prime}$ définie a priori de $D^{\prime}$ dans $\mathbb{R}^{2}$, par

$$
R^{\prime}(\boldsymbol{x})=T_{\phi}(\boldsymbol{x}) \text { si } \boldsymbol{x} \in D_{1}^{\prime} \text {, et } R^{\prime}(\boldsymbol{x})=T_{\phi}(\boldsymbol{x})-(1,0) \text { si } \boldsymbol{x} \in D_{2}^{\prime} \backslash D_{1}^{\prime} .
$$

La suite de la preuve est de vérifier que cet échange de morceaux convient pour certaines valeurs des paramètres $\alpha, \beta$ et $\gamma$. 
L'application $R^{\prime}$ est à valeur dans $D^{\prime}$. Commençons par fixer un élément $\boldsymbol{x}$ de $\psi^{-1}\left(D_{2}\right)$. Il existe donc $(x, y) \in D_{2}$ tel que $\boldsymbol{x}=\psi^{-1}(x, y)=\left(-x / \phi,-y+\alpha x^{2} / \phi^{2}-\beta x / \phi+\gamma\right)$. Nous allons montrer que $T_{\phi}(\boldsymbol{x}) \in \psi^{-1}(D)$.

$$
T_{\phi}(\boldsymbol{x})=\left(\begin{array}{c}
-x / \phi+1 / \phi^{2} \\
-y+\alpha x^{2} / \phi^{2}-\beta x / \phi+\gamma-x / \phi-1 /\left(2 \phi^{3}\right) \\
-\left(x+1 / \phi^{2}-1\right) / \phi \\
-y+\alpha x^{2} / \phi^{2}-\beta x / \phi+\gamma-x / \phi-1 /\left(2 \phi^{3}\right)
\end{array}\right) .
$$

Nous allons vérifier qu'il est possible de fixer les paramètres $\alpha, \beta$ et $\gamma$, afin que $T_{\phi}(\boldsymbol{x})$ soit égal à $\psi^{-1}(R(x, y))$. Calculons :

$$
\begin{aligned}
\psi^{-1}(R(x, y)) & =\psi^{-1}\left(\begin{array}{c}
x+1 / \phi^{2}-1 \\
y+x-1 /\left(2 \phi^{3}\right)
\end{array}\right), \\
& =\left(\begin{array}{c}
-\left(x+1 / \phi^{2}-1\right) / \phi \\
-y-x+1 /\left(2 \phi^{3}\right)+\alpha(x-1 / \phi)^{2} / \phi^{2}-\beta(x-1 / \phi) / \phi+\gamma
\end{array}\right) .
\end{aligned}
$$

Nous voulons donc que l'équation suivante soit satisfaite :

$$
\begin{gathered}
-y+\alpha x^{2} / \phi^{2}-\beta x / \phi+\gamma-x / \phi-1 /\left(2 \phi^{3}\right)=-y-x+1 /\left(2 \phi^{3}\right)+\alpha(x-1 / \phi)^{2} / \phi^{2} \\
-\beta(x-1 / \phi) / \phi+\gamma .
\end{gathered}
$$

Soit encore :

$$
\begin{aligned}
& \alpha x^{2} / \phi^{2}-\beta x / \phi-x / \phi-1 /\left(2 \phi^{3}\right)=-x+1 /\left(2 \phi^{3}\right)+\alpha(x-1 / \phi)^{2} / \phi^{2}-\beta(x-1 / \phi) / \phi, \\
& \Longleftrightarrow\left\{\begin{array}{c}
-1 / \phi=-1-2 \alpha / \phi^{3}, \\
-1 /\left(2 \phi^{3}\right)=1 /\left(2 \phi^{3}\right)+\alpha / \phi^{4}+\beta / \phi^{2}, \\
\alpha=-\phi / 2, \\
\beta=-1 /(2 \phi) .
\end{array}\right.
\end{aligned}
$$

Nous fixons donc pour toute la suite de cette preuve :

$$
\psi(x, y)=\left(-\phi x,-y-\phi x^{2} / 2+\phi^{2} x / 2\right),
$$

d'inverse $\psi^{-1}(x, y)=\left(-x / \phi,-y-x^{2} /(2 \phi)+x /\left(2 \phi^{2}\right)\right)$.

Soit maintenant $\boldsymbol{x} \in D_{1}^{\prime}=T_{\phi}\left(\psi^{-1}\left(D_{1}\right)\right)$. Il existe donc $(x, y) \in D_{1}$ tel que

$$
\boldsymbol{x}=T_{\phi}\left(\psi^{-1}(x, y)\right)=\left(-x / \phi+1 / \phi^{2},-y-x^{2} /(2 \phi)+x /\left(2 \phi^{2}\right)-x / \phi-1 /\left(2 \phi^{3}\right)\right) .
$$

Nous allons montrer que $R^{\prime}(\boldsymbol{x}) \in \psi^{-1}(D)$.

$$
R^{\prime}(\boldsymbol{x})=\left(\begin{array}{c}
-x / \phi+1 / \phi^{2}+1 / \phi^{2}-1 \\
-y-x^{2} /(2 \phi)+x /\left(2 \phi^{2}\right)-x / \phi-1 /\left(2 \phi^{3}\right)-x / \phi+1 / \phi^{2}-1 /\left(2 \phi^{3}\right)
\end{array}\right) .
$$


Pour cela, nous vérifions que $R^{\prime}(\boldsymbol{x})=\psi^{-1}(R(x, y))=\psi^{-1}\left(T_{\phi}(x, y)\right)$ :

$$
\begin{aligned}
\psi^{-1}\left(T_{\phi}(x, y)\right) & =\psi^{-1}\left(x+1 / \phi^{2}, y+x-1 /\left(2 \phi^{3}\right)\right), \\
& =\left(\begin{array}{c}
-x / \phi-1 / \phi^{3} \\
-y-x+1 /\left(2 \phi^{3}\right)-\left(x+1 / \phi^{2}\right)^{2} /(2 \phi)+\left(x+1 / \phi^{2}\right) /\left(2 \phi^{2}\right)
\end{array}\right)
\end{aligned} .
$$

Puisque $-x / \phi+1 / \phi^{2}+1 / \phi^{2}-1=-x / \phi-1 / \phi^{3}$, il ne nous reste qu'à montrer que

$$
\begin{gathered}
-y-x^{2} /(2 \phi)+x /\left(2 \phi^{2}\right)-x / \phi-1 /\left(2 \phi^{3}\right)-x / \phi+1 / \phi^{2}-1 /\left(2 \phi^{3}\right)=-y-x \\
+1 /\left(2 \phi^{3}\right)-\left(x+1 / \phi^{2}\right)^{2} /(2 \phi)+\left(x+1 / \phi^{2}\right) /\left(2 \phi^{2}\right) .
\end{gathered}
$$

Soit encore,

$$
\begin{aligned}
-x^{2} /(2 \phi)-x / \phi-1 /\left(2 \phi^{3}\right)-x / \phi+1 / \phi^{2}-1 /\left(2 \phi^{3}\right)=-x+1 /\left(2 \phi^{3}\right) & -\left(x+1 / \phi^{2}\right)^{2} /(2 \phi)+1 /\left(2 \phi^{4}\right), \\
& \Longleftrightarrow-x^{2} /(2 \phi)-x / \phi-1 /\left(2 \phi^{3}\right)-x / \phi+1 / \phi^{2}-1 /\left(2 \phi^{3}\right)=-x \\
& \left.\Longleftrightarrow-x+1 / \phi^{2}\right)^{2} /(2 \phi)+1 /\left(2 \phi^{2}\right), \\
& \Longleftrightarrow\left\{\begin{array}{ccc}
-2 / \phi & -\left(x+\phi \phi^{3}\right)-x / \phi+1 / \phi^{2}-1 /\left(2 \phi^{3}\right)= & -x-x / \phi^{3}-1 /\left(2 \phi^{5}\right)+1 /\left(2 \phi^{2}\right), \\
-1 / \phi^{3}+1 / \phi^{2} & = & -1 /\left(2 \phi^{5}\right)+1 /\left(2 \phi^{2}\right) .
\end{array}\right.
\end{aligned}
$$

Puisque $\phi^{3}-2 \phi^{2}+1=0$, nous trouvons le résultat attendu. Nous venons donc de montrer que :

$$
T_{\phi}\left(\psi^{-1}\left(D_{1}\right)\right) \subset D_{2}^{\prime}, T_{\phi}\left(\psi^{-1}\left(D_{2}\right)\right) \subset D_{1}^{\prime} \text { et } T_{\phi}\left(D_{2}^{\prime}\right)-(1,0) \subset D_{1}^{\prime} .
$$

Les ensembles $D_{1}^{\prime}$ et $D_{2}^{\prime}$ sont presque sûrement disjoints. Soit $\boldsymbol{x} \in D_{1}^{\prime} \cap D_{2}^{\prime}$. Il existe donc $(x, y) \in D$, et $\left(x_{1}, y_{1}\right) \in D_{1}$, tels que $\boldsymbol{x}=\psi^{-1}(x, y)=T_{\phi}\left(\psi^{-1}\left(x_{1}, y_{1}\right)\right)$. Donc :

$$
\left(\begin{array}{c}
-x / \phi \\
-y-x^{2} /(2 \phi)+x /\left(2 \phi^{2}\right)
\end{array}\right)=\left(\begin{array}{c}
-x_{1} / \phi+1 / \phi^{2} \\
-y_{1}-x_{1}^{2} /(2 \phi)+x_{1} /\left(2 \phi^{2}\right)-x_{1} / \phi-1 /\left(2 \phi^{3}\right)
\end{array}\right) .
$$

Donc $x=x_{1}-1 / \phi$, et

$$
\begin{aligned}
-y & -x^{2} /(2 \phi)+x /\left(2 \phi^{2}\right)=-y_{1}-x_{1}^{2} /(2 \phi)+x_{1} /\left(2 \phi^{2}\right)-x_{1} / \phi-1 /\left(2 \phi^{3}\right), \\
& \Longleftrightarrow y=y_{1}+x_{1}^{2} /(2 \phi)-x_{1} /\left(2 \phi^{2}\right)+x_{1} / \phi+1 /\left(2 \phi^{3}\right)-x^{2} /(2 \phi)+x /\left(2 \phi^{2}\right), \\
& \Longleftrightarrow y=y_{1}+x_{1}^{2} /(2 \phi)-x_{1} /\left(2 \phi^{2}\right)+x_{1} / \phi+1 /\left(2 \phi^{3}\right) \\
& \Longleftrightarrow y=y_{1}+x_{1} / \phi+1 /\left(2 \phi^{3}\right)+x_{1} / \phi^{2}-1 /\left(2 \phi^{3}\right)-1 /\left(2 \phi^{3}\right), \\
& \Longleftrightarrow y=y_{1}+x_{1}-1 /\left(2 \phi^{3}\right) .
\end{aligned}
$$

Nous trouvons $(x, y)=T_{\phi}\left(x_{1}, y_{1}\right)-(1,0)$. Mais puisque $\left(x_{1}, y_{1}\right) \in D_{1}$, le point $\boldsymbol{x}_{0}=T_{\phi}\left(x_{1}, y_{1}\right)$ appartient à l'ensemble $D$. Il existe alors deux points $(x, y)$ et $\boldsymbol{x}_{0}$ de $D$ tels que la différence vaut $(-1,0) \in \mathbb{Z}^{2}$. Donc, $\mu\left(D_{1}^{\prime} \cap D_{2}^{\prime}\right)=0$, ce qui termine cette partie de la preuve.

Il existe un réel $z^{\prime}$ tel que $\left.\left.\mathfrak{p}\left(D_{1}^{\prime}\right) \subset\right] z^{\prime}-1, z^{\prime}+1 / \phi^{2}\right]$ et $\left.\mathfrak{p}\left(D_{2}^{\prime}\right) \subset\right] z^{\prime}-1 / \phi^{2}, z^{\prime}+1 / \phi^{2}[$. C'est immédiat en remarquant qu'en notant $h$ l'homothétie réelle de rapport $-1 / \phi$, alors $\mathfrak{p} \circ \psi^{-1}=h \circ \mathfrak{p}$. 
Donnons-nous deux points $x_{1}$ et $x_{2}$ de $D^{\prime}$ et vérifions que presque sûrement : $x_{1}-x_{2} \notin$ $\mathbb{Z}^{2}$. Commençons par supposer qu'il existe deux points $(x, y) \in D$ et $\left(x_{1}, y_{1}\right)$ de $D_{1}$, et un vecteur entier $\boldsymbol{n}=(n, m) \in \mathbb{Z}^{2}$, tels que $\psi^{-1}(x, y)=T_{\phi} \psi^{-1}\left(x_{2}, y_{2}\right)+(n, m)$. Alors :

$$
\left(\begin{array}{c}
-x / \phi \\
-y-x^{2} /(2 \phi)+x /\left(2 \phi^{2}\right)
\end{array}\right)=\left(\begin{array}{c}
-x_{1} / \phi+1 / \phi^{2}+n \\
-y_{1}-x_{1}^{2} /(2 \phi)+x_{1} /\left(2 \phi^{2}\right)-x_{1} / \phi-1 /\left(2 \phi^{3}\right)+m
\end{array}\right) .
$$

Donc $x=x_{1}-1 / \phi-n \phi$. Puisque $\left.\left.\mathfrak{p}\left(D_{1}\right) \subset\right] z-1, z+1 / \phi^{2}\right]$, et $\left.\left.\mathfrak{p}(D) \subset\right] z-1, z+1+1 / \phi^{2}\right]$, alors nécessairement $n \in\{0,-1\}$.

Si $n=0$, nous pouvons directement utiliser le fait que les ensembles $D_{1}^{\prime}$ et $D_{2}^{\prime}$ sont presque sûrement disjoints. Sinon, $n=-1$, et donc $x=x_{1}+1$. Alors :

$$
\begin{aligned}
-y & -x^{2} /(2 \phi)+x /\left(2 \phi^{2}\right)=-y_{1}-x_{1}^{2} /(2 \phi)+x_{1} /\left(2 \phi^{2}\right)-x_{1} / \phi-1 /\left(2 \phi^{3}\right)+m, \\
& \Longleftrightarrow y=y_{1}+x_{1}^{2} /(2 \phi)-x_{1} /\left(2 \phi^{2}\right)+x_{1} / \phi+1 /\left(2 \phi^{3}\right)-x^{2} /(2 \phi)+x /\left(2 \phi^{2}\right)+m, \\
& \Longleftrightarrow y=y_{1}+x_{1}^{2} /(2 \phi)-x_{1} /\left(2 \phi^{2}\right)+x_{1} / \phi+1 /\left(2 \phi^{3}\right)-\left(x_{1}+1\right)^{2} /(2 \phi) \\
& \Longleftrightarrow y=y_{1}+x_{1} / \phi+1 /\left(2 \phi^{3}\right)-x_{1} / \phi-1 /(2 \phi)+1 /\left(2 \phi^{2}\right)+m, \\
& \Longleftrightarrow y=y_{1}+m .
\end{aligned}
$$

La mesure de ces points est nulle. Nous venons de traiter le cas le plus difficile. En effet, avec les mêmes notations, supposons que $\left(\boldsymbol{x}^{1}, \boldsymbol{x}^{2}\right)$ est un couple de point de $D_{1}^{\prime}$ ou $D_{2}^{\prime}$, tel que $\boldsymbol{x}^{1}-\boldsymbol{x}^{2}=(n, m) \in \mathbb{Z}^{2}$. Alors, avec les contraintes imposées sur les longueurs à $\mathfrak{p}(D)$ et $\mathfrak{p}\left(D_{1}\right)$, il est relativement immédiat que $n=0$. Nous en déduisons alors aisément qu'il existe deux points de $D$ dont la différence est un vecteur de la forme $(0, m)$, où $m$ est un entier. Ces points forment encore un ensemble de mesure nulle.

Vérifions que $\mathcal{L}_{\mathcal{R}^{\prime}}=\mathcal{L}\left(\sigma\left(\mathcal{L}_{\mathcal{R}}\right)\right)$. L'application de premier retour de $R^{\prime}$ dans $D_{1}^{\prime}$ est conjuguée via $\psi$ à $R$, par l'équation 6. Fixons alors $\boldsymbol{x}^{\prime} \in D_{1}^{\prime}$ et notons $\boldsymbol{x}$ le point de $D$, pré-image de $\boldsymbol{x}^{\prime}$ par $\psi$. Nous trouvons ainsi : $C\left(\boldsymbol{x}^{\prime}\right)=\sigma(C(\boldsymbol{x}))$. Supposons maintenant que $\boldsymbol{x}^{\prime} \in D_{2}^{\prime}$ et notons $\boldsymbol{z}^{\prime}=T_{\phi}^{-1} \boldsymbol{x}^{\prime} \in D_{1}^{\prime}$. Par le raisonnement précédent, nous savons qu'il existe $\boldsymbol{x} \in D$, tel que $C\left(\boldsymbol{z}^{\prime}\right)=\sigma(C(\boldsymbol{x}))$. Nous concluons alors en remarquant que $\mathcal{S}\left(C\left(\boldsymbol{z}^{\prime}\right)\right)=C\left(\boldsymbol{x}^{\prime}\right)$ et donc que $C\left(\boldsymbol{x}^{\prime}\right)=\mathcal{S}(\sigma(C(\boldsymbol{x})))$.

Le système symbolique $\mathcal{S}_{\mathcal{R}^{\prime}}$ est conjugué à $\overline{\mathcal{T}}_{\phi}$. Donnons-nous deux points $\boldsymbol{x}^{1}$ et $\boldsymbol{x}^{2}$ de $D^{\prime}$ et supposons qu'ils aient le même codage. Nous reprenons les arguments du point précédent.

Si ces deux points appartiennent à l'ensemble $D_{1}^{\prime}$, alors il existe deux points $\boldsymbol{y}^{1}$ et $\boldsymbol{y}^{2}$ de $D$ tels que $C\left(\boldsymbol{x}^{1}\right)=\sigma\left(C\left(\boldsymbol{y}^{1}\right)\right)$ et $C\left(\boldsymbol{x}^{2}\right)=\sigma\left(C\left(\boldsymbol{y}^{2}\right)\right)$. Puisque la substitution de Fibonacci est un automorphisme du groupe libre : $C\left(\boldsymbol{y}^{1}\right)=C\left(\boldsymbol{y}^{2}\right)$. Nous pouvons conclure car la mesure de ces points est nulle. Nous traitons le cas où les points $\boldsymbol{x}^{1}$ et $\boldsymbol{x}^{2}$ appartiennent à $D_{2}^{\prime}$ de manière analogue.

Preuve de la proposition 5.Q. Notons $z=1 /(2 \phi)+1 /\left(2 \phi^{3}\right)$ et considérons les fonctions quadratiques $p, q$ et $r$, définies pour tout réel $x$, par :

$$
p(x)=\phi^{2} \frac{x^{2}}{2}-\phi \frac{x}{2}-\frac{1}{\phi}, q(x)=p(x)+\phi^{2} x+\frac{3}{2} \text { et } r(x)=p(x)-\phi^{2} x+1+\frac{1}{2 \phi^{3}} .
$$


Nous définissons les zones $D_{1}^{(1)}$ et $D_{2}^{(1)}$ par :

$$
\begin{aligned}
D_{1}^{(1)} & =\{(x, y) ; p(x)<y \leq p(x)+1 \text { et } y \leq \min (q(x), r(x)-1)\} \\
\text { et } \quad D_{2}^{(1)} & =\{(x, y) ; p(x)<y \leq p(x)+1 \text { et } r(x)-1<y \leq r(x)\} .
\end{aligned}
$$

Nous notons $R^{(1)}$ l'application de l'ensemble $D$ dans lui-même définie par

$$
R^{(1)}(\boldsymbol{x})=T_{\phi}(\boldsymbol{x}) \text {, si } \boldsymbol{x} \in D_{1} \text {, et } R^{(1)}(\boldsymbol{x})=T_{\phi}(\boldsymbol{x})-(1,0) \text { si } \boldsymbol{x} \in D_{2} .
$$

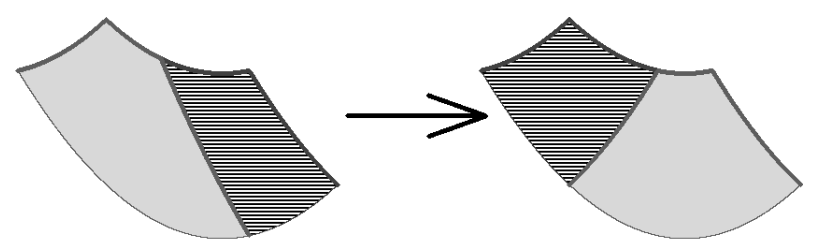

FiguRE 1 - Représentation de l'application $R^{(1)} \operatorname{sur} D^{(1)}=D_{1}^{(1)} \cup D_{2}^{(1)}$.

Nous montrons alors que le système symbolique $\mathcal{S}_{\mathcal{R}}$ est conjugué à $\overline{\mathcal{T}}_{\phi}$ en utilisant la proposition 3.1. Soit $\boldsymbol{x}^{1}=\left(x_{1}, y_{1}\right)$ et $\boldsymbol{x}^{2}=\left(x_{2}, y_{2}\right)$ deux points de $D^{(1)}$ tels que $C\left(\boldsymbol{x}^{1}\right)=C\left(\boldsymbol{x}^{2}\right)$. Commençons par supposer que $x_{1}=x_{2}-\delta$ avec $\delta>0$. Notons $\mathfrak{p}$ la projection $(x, y) \mapsto x$ et remarquons que pour tout couple $\left(\boldsymbol{y}, \boldsymbol{y}^{\prime}\right)$ de $D_{1}^{(1)}$ (respectivement $\left.D_{2}^{(1)}\right), \mathfrak{p}(\boldsymbol{y})-\mathfrak{p}\left(\boldsymbol{y}^{\prime}\right)=\mathfrak{p}\left(R^{(1)} \boldsymbol{y}\right)-\mathfrak{p}\left(R^{(1)} \boldsymbol{y}^{\prime}\right)$. Donc si $C\left(\boldsymbol{x}^{1}\right)=C\left(\boldsymbol{x}^{2}\right)$,

$$
\text { pour tout entier } n, \mathfrak{p}\left(R^{(1)^{n}}\left(\boldsymbol{x}^{2}\right)\right)-\mathfrak{p}\left(R^{(1)^{n}}\left(\boldsymbol{x}^{1}\right)\right)=\delta \text {. }
$$

Par minimalité du système $\overline{\mathcal{T}}_{\phi}$, il existe un entier $n_{0}$ tel que :

$$
\left.R^{(1)^{n_{0}}}\left(\boldsymbol{x}^{2}\right) \in\right] z-1 /\left(\phi^{2}\right), z-1 /\left(\phi^{2}\right)+\delta / 2\left[\times \mathbb{R} \cap D_{2}^{(1)} .\right.
$$

Nous arrivons à une contradiction puisque $\left\{z-1 /\left(\phi^{2}\right)-\delta / 2\right\} \times \mathbb{R} \cap D_{2}^{(1)}=\emptyset$, donc nécessairement $x_{1}=x_{2}$. Nous allons appliquer le même raisonnement pour montrer que $y_{1}=y_{2}$. Notons $x=$ $x_{1}=x_{2}$, et supposons que $y_{1}=y_{2}+\delta$, où $\delta>0$. Notons $\mathfrak{q}$ la projection $(x, y) \mapsto y$. Comme précédemment, si $C\left(\boldsymbol{x}^{1}\right)=C\left(\boldsymbol{x}^{2}\right)$,

$$
\text { pour tout entier } n, \mathfrak{q}\left(R^{(1)^{n_{0}}}\left(\boldsymbol{x}^{1}\right)\right)-\mathfrak{q}\left(R^{(1)^{n}}\left(\boldsymbol{x}^{2}\right)\right)=\delta .
$$

Il existe $\epsilon>0$, et un entier $n_{0}$ tels que

$$
\left.R^{(1)^{n_{0}}}\left(\boldsymbol{x}^{1}\right) \in\right] z, z-\epsilon\left[\times \mathbb{R} \cap D_{1}^{(1)},\right.
$$

et donc l'ensemble $] z, z-\epsilon+\delta\left[\times \mathbb{R} \cap D^{(1)}+(0, \delta)\right.$ est disjoint de $D_{1}^{(1)}$.

Preuve du théorème 2.2. Nous avons déjà vu dans la proposition 3.2 que pour tout entier $M$, $p^{T_{\phi}}(M) \geq M+1$. Nous allons montrer que pour tout entier $M$ fixé, nous pouvons construire un échange de morceaux $\mathcal{R}^{(N)}$ conjugué à $T_{\phi}$, tel que pour tout entier $1 \leq k \leq M, p_{\mathcal{R}^{(N)}}(k)=k+1$. 
Nous définissons pour tout entier $N$ par récurrence un échange de morceaux $\mathcal{R}^{(N)}$ conjugué à $T_{\phi}$ et satisfaisant les hypothèses de la proposition 5.1. Nous initialisons la récurrence en considérant l'échange de morceaux $\mathcal{R}^{(1)}$ construit dans la proposition 5.2. Le $(N+1)$-ième échange de morceaux est celui obtenu par la proposition 5.1 appliquée à l'échange de morceaux $\mathcal{R}^{(N)}$.

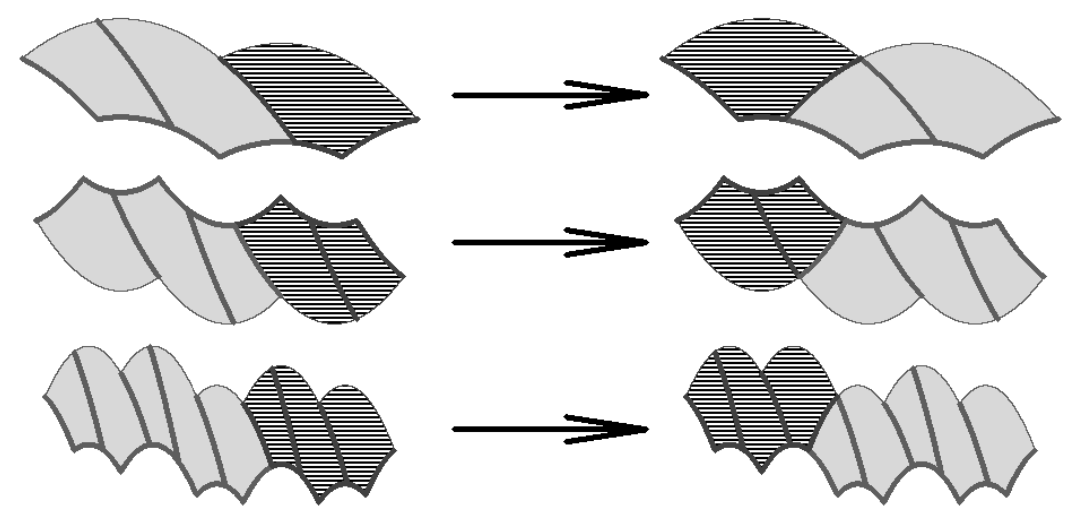

FiguRE 2 - Représentation des échanges de morceaux $\mathcal{R}^{(2)}, \mathcal{R}^{(3)}$ et $\mathcal{R}^{(4)}$.

Commençons donc par fixer un entier $M$. Nous notons $\mathfrak{L}^{\phi}$ le langage factoriel généré par le mot de Fibonacci, point fixe de la substitution $\sigma$. Par l'équation (5) et le lemme suivant, la suite de langages $\left(\mathfrak{L}_{\mathcal{R}^{(N)}}\right)_{N}$ associés aux échanges de morceaux $\left(\mathcal{R}^{(N)}\right)_{N}$ converge au sens de l'équation (8) vers le langage $\mathfrak{L}^{\phi}$, qui est de complexité $M \mapsto M+1$.

Nous énonçons maintenant un lemme combinatoire dont la preuve nous a été communiquée par J. Cassaigne.

Lemme 5.3. Soit $\sigma$ la substitution de Fibonacci définie en (4). Nous notons $\mathfrak{L}^{\phi}$ le langage factoriel généré par le mot de Fibonacci, point fixe de la substitution $\sigma$. Nous définissons pour tout langage factoriel $\mathfrak{L}$ de $\{1,2\}^{*}$ une suite de langages $\left(\mathfrak{L}_{N}\right)_{N}$ par récurrence de la manière suivante:

$$
\mathfrak{L}_{0}=\mathfrak{L} \text { et pour tout entier } n \geq 0, \mathfrak{L}_{N+1}=\mathcal{L}\left(\sigma\left(\mathfrak{L}_{N}\right)\right) .
$$

Alors la suite de langages $\left(\mathfrak{L}_{N}\right)_{N}$ converge vers $\mathfrak{L}^{\phi}$, dans le sens où pour tout entier $M$, il existe un rang $N_{0}$ à partir duquel pour tout $N \geq N_{0}$,

$$
\left\{u \in \mathfrak{L}_{N} \text { de longueur inférieure } \grave{a} M\right\}=\left\{u \in \mathfrak{L}^{\phi} \text { de longueur inférieure à } M\right\} .
$$

Preuve du lemme 5.3. Donnons-nous deux langages $\mathfrak{L}$ et $\mathfrak{L}^{\prime}$, et associons-leur les suites de langages définis en (7), respectivement notées $\left(\mathfrak{L}_{N}\right)_{N}$ et $\left(\mathfrak{L}_{N}^{\prime}\right)_{N}$. Nous commençons par remarquer que si $\mathfrak{L}$ est inclus dans $\mathfrak{L}^{\prime}$, alors pour tout entier $N$ le langage $\mathfrak{L}_{N}$ est inclus dans $\mathfrak{L}_{N}^{\prime}$. Il suffit donc de traiter les deux cas extrêmes : $\mathfrak{L}=\{1,2, \epsilon\}$ et $\mathfrak{L}=\{1,2\}^{*}$.

Dans le premier cas, la suite $\left(\mathfrak{L}_{N}\right)_{N}$ est croissante (pour l'inclusion); la limite des langages $\left(\mathfrak{L}_{N}\right)_{N}$ est donc leur réunion qui est clairement le langage $\mathfrak{L}^{\phi}$ du mot de Fibonacci.

Dans le second cas, la suite de langages $\left(\mathfrak{L}_{N}\right)_{N}$ est décroissante et sa limite est son intersection qui contient clairement le langage $\mathfrak{L}^{\phi}$. L'inclusion réciproque est plus délicate : c'est le seul endroit où nous utilisons des propriétés spécifiques de la substitution de Fibonacci. Si $w$ est dans l'intersection 
des langages $\left(\mathfrak{L}_{N}\right)_{N}$, alors en particulier $w$ est facteur de mots de la forme $\sigma^{m}\left(v_{m}\right)$, où $m$ est un entier et $v_{m}$ un mot quelconque. Nous pouvons alors supposer que $m$ est un entier non nul, tel que $\left|s^{m}(1)\right| \geq\left|s^{m}(2)\right| \geq|w|$ et que $v_{m}$ un mot de longueur au plus 2. Si $v_{m}$ n'est pas le mot $22, v_{m}$ est dans le langage $\mathfrak{L}^{\phi}$ (qui contient clairement les mots 11, 12 et 22 ) donc $w$ aussi. Si $v_{m}=22$, alors $w$ est facteur de $\sigma^{m}(22)=\sigma^{m-1}(11)$ : il est donc également un mot du langage $\mathfrak{L}^{\phi}$.

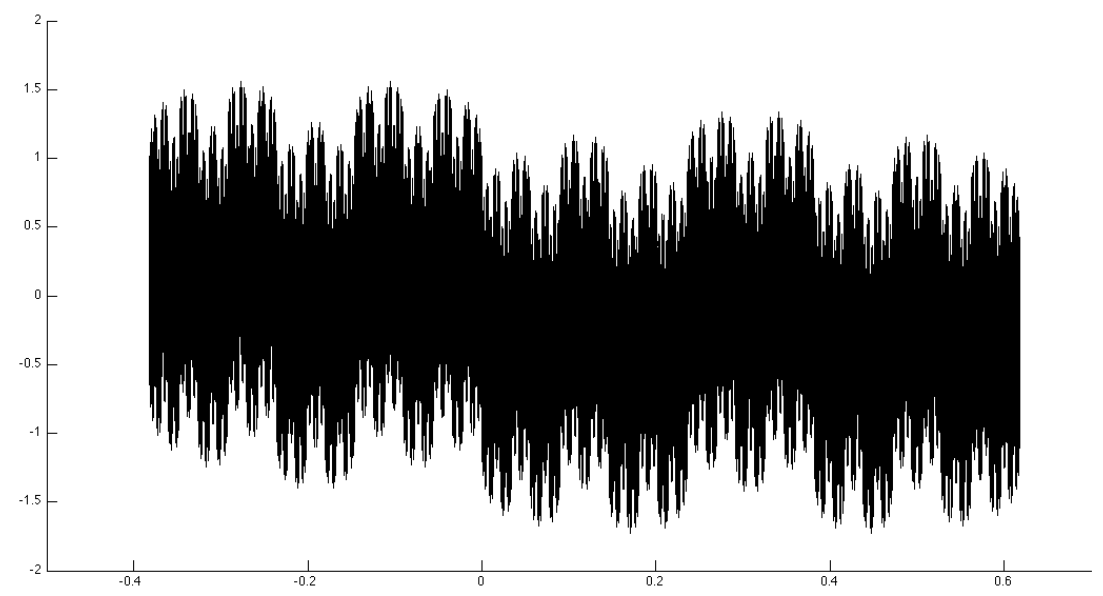

Figure 3 - Représentation du domaine $D^{(20)}$.

Remarque 1. Expliquons pourquoi la suite d'échanges de morceaux $\left(\mathcal{R}^{(N)}\right)_{N}$ ne converge pas vers un «échange de morceaux bornés limite».

Si cet échange de morceaux limite $\mathcal{R}^{(\infty)}$ existait, il serait un échange affine de deux morceaux $D_{1}^{(\infty)}$ et $D_{2}^{(\infty)}$ dont l'image par la projection $\mathfrak{p}(x, y)=x$ serait respectivement $[-1 / \phi, 0]$ et $] 0,1 / \phi^{2}[$.

Pour tout point $\boldsymbol{x}_{0}=\left(x_{0}, y_{0}\right)$ de $D^{(\infty)}=D_{1}^{(\infty)} \cup D_{2}^{(\infty)}$, nous nous intéressons à la suite de points $\left(\boldsymbol{x}_{n}\right)_{n}$ définie par recurrence par $\boldsymbol{x}_{n+1}=\mathcal{R}^{(\infty)}\left(\boldsymbol{x}_{n}\right)$.

Si nous notons $\{z\}$ la partie fractionnaire d'un nombre réel $z$, alors par construction de la suite d'échanges de morceaux $\left(\mathcal{R}^{(N)}\right)_{N}$ construits dans la preuve du théorème 2.2 nous trouvons :

$$
\begin{aligned}
\boldsymbol{x}_{n} & =\left(\left\{x_{0}+\frac{n}{\phi^{2}}\right\}-\frac{1}{\phi}, y_{0}+\frac{n}{2 \phi^{3}}+\sum_{k=0}^{n}\left(\left\{x_{0}+\frac{k}{\phi^{2}}\right\}-\frac{1}{\phi}\right)\right), \\
& =\left(\left\{x_{0}+\frac{n}{\phi^{2}}\right\}-\frac{1}{\phi}, y_{0}+\sum_{k=0}^{n}\left(\left\{x_{0}+\frac{k}{\phi^{2}}\right\}-\frac{1}{2}\right)\right) .
\end{aligned}
$$

Par le travail de C.G. Pinner dans [5],

$$
\underset{N}{\limsup }\left|\frac{n}{2}-\sum_{k=0}^{n}\left\{x_{0}+\frac{k}{\phi^{2}}\right\}\right|=+\infty .
$$

Donc le domaine $D^{(\infty)}$ ne serait pas borné. 


\section{Références}

[1] P. Arnoux et C. Mauduit, 'Complexité de suites engendrées par des récurrences unipotentes', Acta Arithmetica, 76 (1996) 1, 85-97.

[2] P. Arnoux, C. Mauduit, L. Shiokawa et J.-I.Tamura, 'Rauzy's conjecture on billiards in the cube', Tokyo Journal of Mathematics, 17 (1994) 1, 211-218.

[3] P. Arnoux, C. Mauduit, L. Shiokawa et J.-I.Tamura, 'Complexity of sequences defined by billiard in the cube', Bulletin de la Société Mathématique de France, 122 (1994) 1, 1-12.

[4] V. Berthé, S. Ferenczi, et L.Q. Zamboni, 'Interactions between dynamics, arithmetics and combinatorics : the good, the bad, and the ugly', Algebraic and topological dynamics, Contemp. Math., 385 (2005), 333-364.

[5] C.G. Pinner, 'On the one-sided boundedness of sums of fractional parts $\left(\{n \alpha+\gamma\}-\frac{1}{2}\right)$ ', Journal of Number Theory, 81, (2000) 1, 170-204.

[6] G. Rauzy, 'Des mots en arithmétique', Avignon conference on language theory and algorithmic complexity (Avignon, 1983), Publ. Dép. Math. Nouvelle Sér. B, 84 (1984) 103-113. 